\title{
Shen Shuai IIRecipe attenuates renal injury and fibrosis in chronic kidney disease by regulating NLRP3 inflammasome and Sirt1/ Smad3 deacetylation pathway
}

Meng Wang ${ }^{1,2}$, Liuyi Yang ${ }^{1,2}$, Jing Yang ${ }^{1,2}$ and Chen Wang ${ }^{1,2,3,4^{*}}$ (D)

\begin{abstract}
Background: Excessive activation of NLRP3 inflammasome and down-regulation of Sirt1/Smad3 deacetylation pathway play a significant role in the evolution of renal fibrosis. In China, it has been well known that Chinese herbal medicine is markedly effective in treating chronic kidney disease (CKD). Shen Shuai IIRecipe (SSR) has been used clinically for more than 20 years and has been confirmed to be effective in improvements of renal function and fibrosis. However, the specific mechanisms under the efficacy require further research. The purpose of this study was to evaluate whether SSR could alleviate renal injury and fibrosis by regulating NLRP3 inflammasome and Sirt1/Smad3 deacetylation pathway.
\end{abstract}

Methods: Four weeks after 5/6 ablation/infarction (A/I) surgery, Sprague-Dawley rats were randomly divided into the following groups: sham operation group, 5/6 (A/I) group, 5/6 (A/I) + SSR group, and 5/6 (A/I) + Losartan group $(5 / 6(A / I)+$ Los). After 8 weeks intervention,we mainly assessed the severity of renal injury and fibrosis along with the activation of NLRP3 inflammasome and Sirt1/Smad3 deacetylation pathway.

Results: SSR significantly attenuated renal injury and fibrosis in the remnant kidneys. In addition, we found that SSR effectively inhibited activation of NLRP3/ASC/Caspase-1/IL-1ßcascade, decreased inflammatory infiltration and upregulated Sirt1/Smad3 deacetylation pathway.

Conclusions: SSR could contribute to renal protection by inhibiting the activation of NLRP3 inflammasome and, furthermore, strengthen the antifibrotic effects by up-regulating Sirt1/Smad3 deacetylation pathway in 5/6 renal (A/I) model.

Keywords: Shen Shuai IIRecipe,Chronic kidney disease, NLRP3 inflammasome, Renal fibrosis, Deacetylation

\section{Background}

Chronic kidney disease (CKD) affects over $10 \%$ of the human population worldwide with high mortality, in part due to limited available or affordable treatment [1]. The prevalence of CKD is increasing worldwide and renal fibrosis is the common outcome of CKD, irrespective of the initial causes [2,3]. The activation of renal fibroblasts and excessive accumulation of

\footnotetext{
*Correspondence: chenwang42@126.com

'Department of Nephrology, Shuguang Hospital Affiliated to Shanghai University of Traditional Chinese Medicine, Shanghai 201203, China

${ }^{2}$ Key Laboratory of Liver and Kidney Diseases,Ministry of Education, Shanghai

University of Traditional Chinese Medicine, Shanghai 201203, China

Full list of author information is available at the end of the article
}

extracellular matrix (ECM) components are the hallmark of renal tubulointerstitial fibrosis,which is closely interconnected with tissue regeneration and inflammation [4].

Tubulointerstitial inflammation is a critical pathological feature of CKD and promotes the evolution of interstitial fibrosis [5].NLRP3,a NOD-like pattern recognition receptor,initiates innate immune response during tissue injury or pathogen infections [6]. Upon activation,the NLRP3 proteins undergo oligomerization and recruit the adaptor protein ASC and pro-caspase- 1 to form inflammasome [7, 8]. The formation of the inflammasome facilitates the release of active caspase- 1 and

(c) The Author(s). 2019 Open Access This article is distributed under the terms of the Creative Commons Attribution 4.0 International License (http://creativecommons.org/licenses/by/4.0/), which permits unrestricted use, distribution, and reproduction in any medium, provided you give appropriate credit to the original author(s) and the source, provide a link to the Creative Commons license, and indicate if changes were made. The Creative Commons Public Domain Dedication waiver (http://creativecommons.org/publicdomain/zero/1.0/) applies to the data made available in this article, unless otherwise stated. 
the subsequent secretion of mature pro-inflammatory cytokines interleukin (IL)-1 $\beta$ and IL-18.Previous reports suggested the NLRP3 complex could play a significant role in promoting inflammatory response and renal fibrosis in CKD [9]. Zhuang et al. [10] demonstrated activation of NLRP3 inflammasome induced by proteinuria was involved in the progressive loss of renal function in CKD. Ludwig-Portugall et al. [11]. confirmed that CP-456,773,a specific inhibitor of the NLRP3 inflammasome, attenuated crytal-induced kidney fibrosis.

Transforming growth factor $\beta-1$ (TGF $\beta-1$ ),a profibrotic factor, has been shown to implicate in activation of renal fibroblasts and accumulation of ECM proteins [12].TGF- $\beta 1$ signal activates Smad2 and Smad3 phosphorylation and subsequently forms a complex with Smad4 to modulate the transcription of target genes [13, 14].However,previous studies reported acetylation of Smad3 was involved in TGF $\beta 1$-dependent renal fibrosis [15]. Sirtuin 1(Sirt1),a conserved $\mathrm{NAD}^{+}$-dependent protein deacetylase, is a survival factor that is involved in lifespan extension and has been demonstrated to deacetylate the lysine residues [16]. He et al. [17].confirmed that activation of Sirt1 attenuated kidney fibrosis in UUO model. Therefore, the inhibition of NLRP3 inflammasome and activation of Sirt1 could be attractive targets to ameliorate renal fibrosis.

Traditional Chinese medicine (TCM) has been widely used for disease prevention and treatment over many years of clinical validation and become popular for promoting healthcare [18]. In China, it has been well known that Chinese herbal medicine is markedly effective in treating CKD. Shen Shuai IIRecipe (SSR),an effective prescription for the treatment of CKD in the clinic, is composed of Epimedium,Codonopsis pilosula, Salvia miltiorrhiza bge, Rheum palmatum, Angelica sinensis, Folium perillae, Coptis chinensis, Peach kernel,and Ligusticum chuanxiong, aiming to'fortify the spleen and tonify the kidney'and'activate blood and resolve stasis'.Clinically, SSR has been used for more than 20 years and become the basic prescription for the treatment of patients with CKD. Previous clinical studies suggested SSR significantly improved renal function of patients with CKD, as evidenced by decreased levels of serum creatinine (Scr),24-h urinary protein quantity, blood urea nitrogen (BUN) and increased evaluated glomerular filtration rates (eGFRs) [19]. Renal ultrasound detection revealed that SSR markedly increased renal blood flow in primary CKD 3 or 4 stage patients [19]. Previous experimental studies showed that SSR significantly down-regulated the expression of NF- $\mathrm{KB} / \mathrm{TNF}-\alpha$ signaling pathway and attenuated renal fibrosis by improving oxygen consumption in 5/6 renal ablation/infarction (A/ I) model $[20,21]$. Importantly, We didn't observe any side effects or changes of liver function in clinical patients and experimental animals. These findings may indicate that the therapeutic effect of SSR is devoid of any toxic manifestation. In the present study, we aimed to investigate whether SSR could alleviate renal fibrosis by regulating NLRP3 inflammasome and Sirt1/Smad3 deacetylation pathway.

\section{Methods}

\section{Animals and drugs}

Eight-week-old male Sprague-Dawley (SD) rats (190 g$210 \mathrm{~g}$ ) were obtained from SLAC Laboratory Animal Co., Ltd. (Shanghai,China) and maintained under a controlled temperature $\left(23 \pm 3^{\circ} \mathrm{C}\right)$ and humidity $(55 \pm 15 \%)$ with a $12 \mathrm{~h}$ light $/ 12 \mathrm{~h}$ dark cycle. SSR consists of Epimedium $15 \mathrm{~g}$,Codonopsis pilosula $15 \mathrm{~g}$,Salvia miltiorrhiza bge $15 \mathrm{~g}$,Rheum palmatum $15 \mathrm{~g}$,Angelica sinensis $15 \mathrm{~g}$,Folium perillae $15 \mathrm{~g}$,Coptis chinensis $6 \mathrm{~g}$,Peach kernel $15 \mathrm{~g}$,and Ligusticum chuanxiong $15 \mathrm{~g}$. The raw herbs for preparation of SSR were obtained from Shanghai Kangqiao Chinese Medicine Tablet Co.,Ltd. (Shanghai,China) and identified by Dr. Guanglin Xu from Pharmacy Department, Shuguang Hospital Affiliated to Shanghai University of TCM. The above nine commonly used Chinese herbs were mixed with distilled water in proportion and heated twice at $100{ }^{\circ} \mathrm{C}$ for $1 \mathrm{~h}$ under continuous stirring condition. The aqueous extracts were subjected to centrifugation at $1500 \times \mathrm{g}$. The supernatants were merged and concentrated to prepare suspension containing $6 \mathrm{~g} / \mathrm{mL}$ of the original drug by means of ethanol. The gavage dose $(10 \mathrm{~mL} / \mathrm{kg})$ in the present study was determined according to the previous studies [21]. Losartan tablets $(100 \mathrm{mg} /$ tablet $)$ were obtained from MSD Pharmaceutical Co., Ltd.(Hangzhou,China) and used as positive control. Losartan was dissolved and diluted by saline with a concentration of $5 \mathrm{mg} / \mathrm{mL}$ of solution.

\section{Rat 5/6(A/I) model and animal study protocol}

$5 / 6$ (A/I) surgery was performed as described previously $[21,22]$. The rats were anesthetized with sodium pentobarbital $(40 \mathrm{mg} / \mathrm{kg}$, i.p.), and placed on temperature-controlled surgical table. The surgical area was disinfected with $75 \%$ alcohol and a flank incision was then performed. The left kidney was taken out from the retroperitoneum and the renal artery of left kidney was exposed after separation of perirenal fat and renal capsular. Two branches of the left renal artery were ligated with 4-0 silk sutures and the gelatin sponge was used to suppress the bleeding for a moment. The left kidney was gently retracted back into the body and the incision was sutured. After one week,a right flank incision was performed. After separating the adrenal gland, the right renal pedicle was ligated and the kidney was removed. 45 rats were randomized into three groups after 4 weeks and administered SSR $(10 \mathrm{~mL} / \mathrm{kg}$ daily by gavage, $n=15)$,losartan $(6 \mathrm{~mL} / \mathrm{kg}$ daily by gavage, $\mathrm{n}=15)$ or 
saline treatment. The sham group underwent the same anesthesia and procedures consisting of manipulation of the perirenal fat and renal pedicles, except for the destruction of renal tissue. A group of 15 rats that received sham operation were also included in the study. After 8 weeks intervention,all animals were anaesthetized with sodium pentobarbital $(40 \mathrm{mg} / \mathrm{kg})$ administered intraperitoneally and euthanized by cervical dislocation. The remnant kidney tissues were collected for molecular examination. The animal procedures were approved by the Animal Experiment Ethics Committee of Shanghai University of Traditional Chinese Medicine in accordance with the principles outlined in the NIH Guide for the Care and Use of Laboratory Animals.

\section{Immunoprecipitation analysis}

Kidney tissues were lysed on ice for $15 \mathrm{~min}$ in lysis buffer containing protease inhibitor. Approximately $500 \mu \mathrm{g}$ of total protein was incubated overnight at $4{ }^{\circ} \mathrm{C}$ with anti-Smad3(Santa Cruz,USA) followed by precipitation with $70 \mu \mathrm{l}$ of protein A/G-Plus-Agarose (Santa Cruz,USA) for $4 \mathrm{~h}$ at $4{ }^{\circ} \mathrm{C}$.Non-specific IgG (Proteintech) was used as negative control. The precipitated complexes were washed in IP buffer and then, resuspended in $30 \mu \mathrm{l}$ of $2 \times$ loading buffer and boiled for 5 min.

\section{Western blot}

The proteins were isolated from the frozen kidneys and the concentration was calculated by the Bradford method. Immunoblotting examination was performed as previously described [21]. In this study, the primary antibodies used were anti-Ecadherin (1:1000,Abcam,UK), anti-ColIII (11,000, Abcam, UK),anti-caspase1. (1:1000, Abcam,UK), anti-TGFßR1 (1:1000,Abcam,UK), anti-Sirt1 (11,000, Abcam, UK), anti-NLRP3 (1:1000, Abclonal, China), anti-IL-1 $\beta \quad$ (11,000,Abclonal,China), anti-Acetylated-lysine (1:500,Santa Cruz,USA), anti-ASC (1:500, Santa Cruz, USA), anti-TGF- $\beta 1$ (1500,Santa Cruz,USA), anti-Smad3 (1,1000,CST,USA), and anti-Gapdh $(12,000$, Proteintech, USA). Protein bands were visualized using the chemiluminescence system (Tanon) for the required time. Quantitative analysis was performed using ImageJ software.

\section{Histology staining and evaluation}

Paraffin-embedded kidneys were sectioned into $3 \mu \mathrm{m}$ slices and subjected to Masson's trichrome and periodic acid-Schiff (PAS) staining according to the standard protocol. For PAS-stained sections, the severity of glomerular injury was assessed in 50 glomeruli using the glomerulosclerosis (GS) score from 0 to 4 and the mean score was calculated [23]. The severity of tubulointerstitial fibrosis was calculated by the Masson's trichrome-positive areas in 4 randomly selected fields per section of kidney using Image-Pro Plus 6.0.

\section{Immunohistochemical (IHC) and immunofluorescence (IF) staining}

IHC staining was performed as described previously [21]. In this study, the primary antibodies used were anti-Ecadherin (1200,Abcam) and anti-Sirt1 (1200, Abcam). Analysis of semi-quantification was performed in 4 randomly selected fields per section of kidney using Image-Pro Plus 6.0.For immunofluorescence staining,antigen was retrieved by microwaveEDTA buffer antigen retrieval method and blocked with $3 \%$ BSA and $0.1 \%$ Triton-100 after quenching autofluorescence. The sections were incubated with anti- $\alpha$ SMA (1200,Abcam,UK) and anti-F4/80(1200,Servicebio, China) overnight at $4{ }^{\circ} \mathrm{C}$.The following secondary antibodies were used: FITC-labeled goat anti-rabbit IgG (Beyotime,China) and Cy3-labeled goat anti-mouse IgG (Beyotime,China). Positive staining was observed by fluorescence microscopy (Nikon Eclipse $80 \mathrm{i}$, Japan) at $400 \times$ magnification.

\section{Statistical analysis}

All results were expressed as mean \pm standard deviation (SD) and analyzed by one-way analysis of variance (ANOVA) with LSD-t's multiple comparisons, using SPSS version 18.0 software. $P<0.05$ was considered statistically significant.

\section{Results}

SSR attenuated renal injury and fibrosis in 5/6(A/I) model In previous studies,we confirmed SSR significantly attenuated the expression of Collagen-I (Col-I) and fibronectin (FN) proteins,two major ECM proteins, as well as $\alpha$-smooth muscle actin ( $\alpha$-SMA) protein,the hallmark of fibroblast activation [21]. Based on the previous results, the study aimed to further assesse the expression of Collagen-III (Col-III) and E-cadherin proteins. Immunoblotting examination showed the expression of Col-III and loss of the epithelial cell marker E-cadherin were increased in the remnant kidneys (Fig. 1(a) and (b)).The IHC analysis of E-cadherin expression showed the same pattern as that of immunoblotting in 5/6(A/I) group (Fig. 1(c) and (d)). Conversely,SSR treatment markedly increased E-cadherin expression and decreased Col-III expression at protein levels in 5/6 (A/I) rats (Fig. 1(a),(b),(c) and(d)).

Consistent with above observations, histopathological examination confirmed SSR showed significant reduction in interstitial fibrosis and renal injury when compared with model group (Fig. 1(e),(f),(g)and1(h)).SSR treatment for 8 weeks displayed less tubular injury, tubulointerstitial inflammation, and fibrosis after 5/6(A/ 

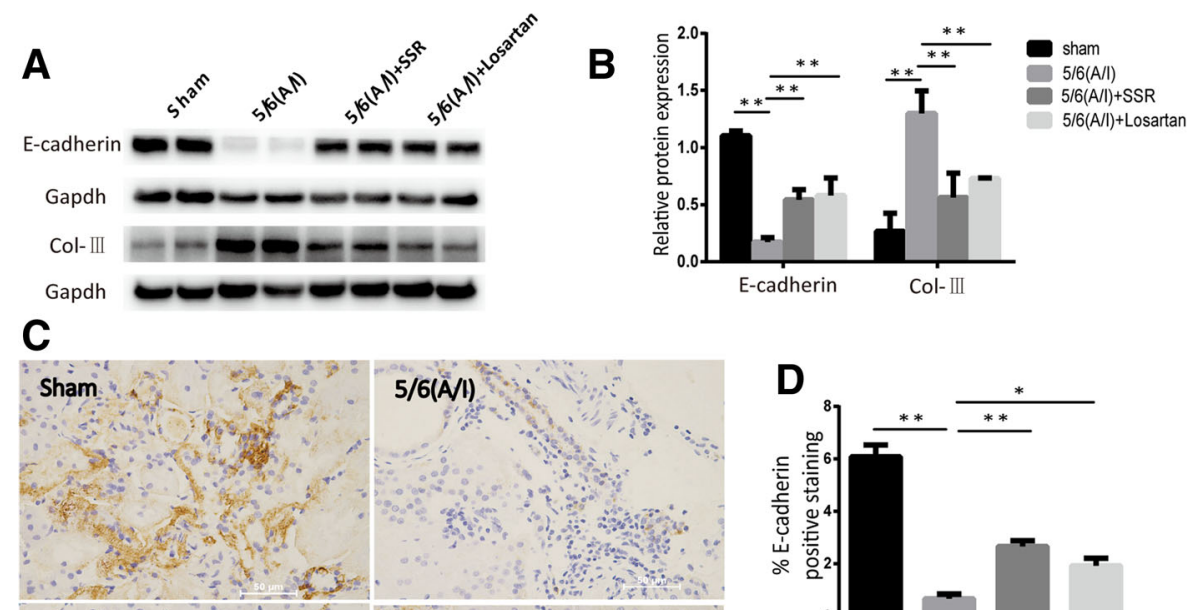

\section{$5 / 6(A /)$}

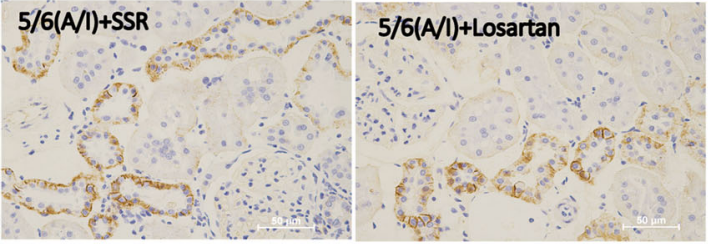

E

PAS

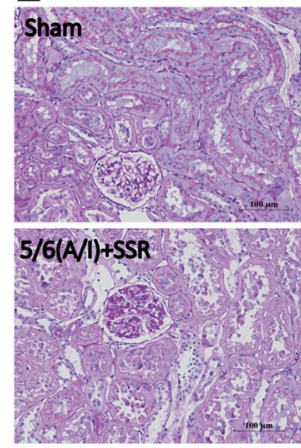

G

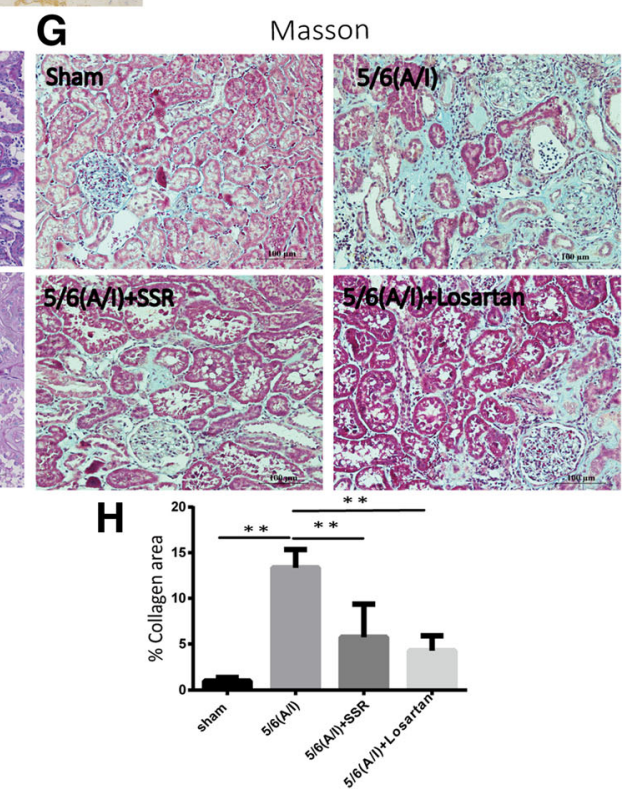

$\mathbf{F}$

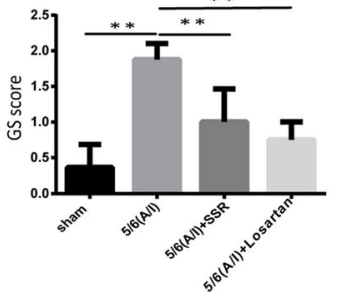

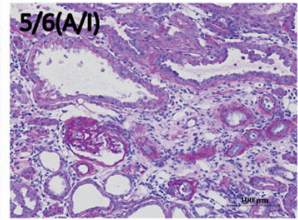

$5 / 6(\mathrm{~A} /)+$ Losartan
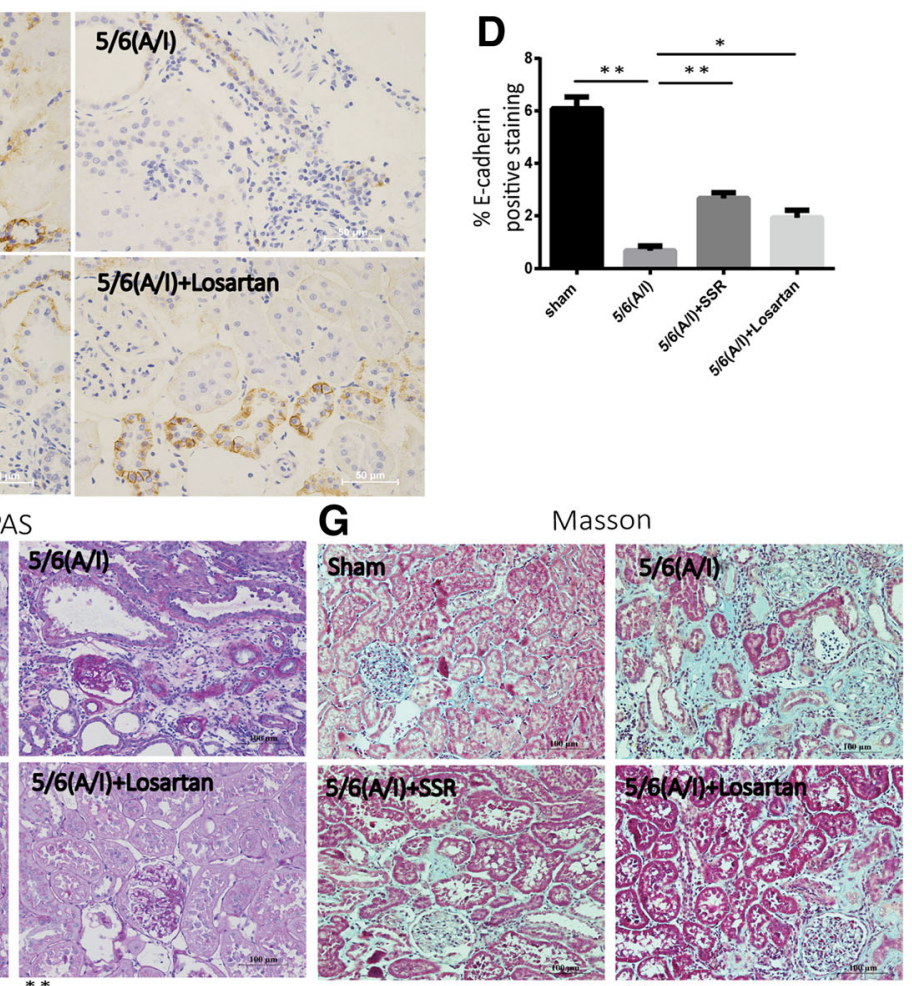

Masson

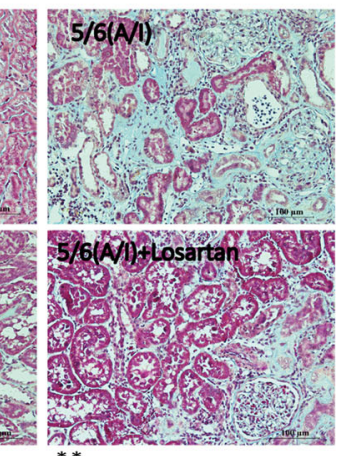

Fig. 1 SSR attenuated renal injury and fibrosis in 5/6(A/I) model. (a) Immunoblot analysis of E-cadherin and Col-IIlexpression. (b) Western blot quantification of E-cadherin and Col-III levels. $(n=6)(\mathbf{c})$ Representative images of E-cadherin expression detected by IHC. $400 \times$ magnification. (d) Quantitative analysis of E-cadherin positive $\operatorname{area}(n=4)$ (e) Representative photomicrographs of PAS staining. $200 \times$ magnification. (f) The severity of glomerular injury was assessed using the glomerulosclerosis (GS) score (g) Assessment of interstitial fibrosis by Masson's trichrome staining.200 $\times$ magnification. (h) Semiquantitative result of collagen area( $\mathrm{n}=4)$. Values are mean \pm SD. ${ }^{*} P<0.05$, ${ }^{*} P<0.01$

I) operation,verifying a protective effect for SSR in renal injury (Fig. 1(e),(f), (g)and1(h)).

SSR inhibited NLRP3 inflammasome activation and IL-1 $\beta$ secretion in $5 / 6(\mathrm{~A} / \mathrm{I})$ rats.

Inflammation plays an important role in the development of renal fibrosis [24]. Renal NLRP3 inflammasome activation characterized by the elevation of
NLRP3,ASC and Caspase-1 activation at protein levels was observed in the remnant kidneys with CRF,which was restored by SSR (Fig. 2(a) and2(b)).Results of inflammasome activation led to secretion of proinflammatory cytokine IL-1 $\beta$ (Fig. 2(c) and (d)).Consistent with above results, SSR decreased protein levels of mature IL- $1 \beta$ compared with $5 / 6(\mathrm{~A} / \mathrm{I})$ operation 


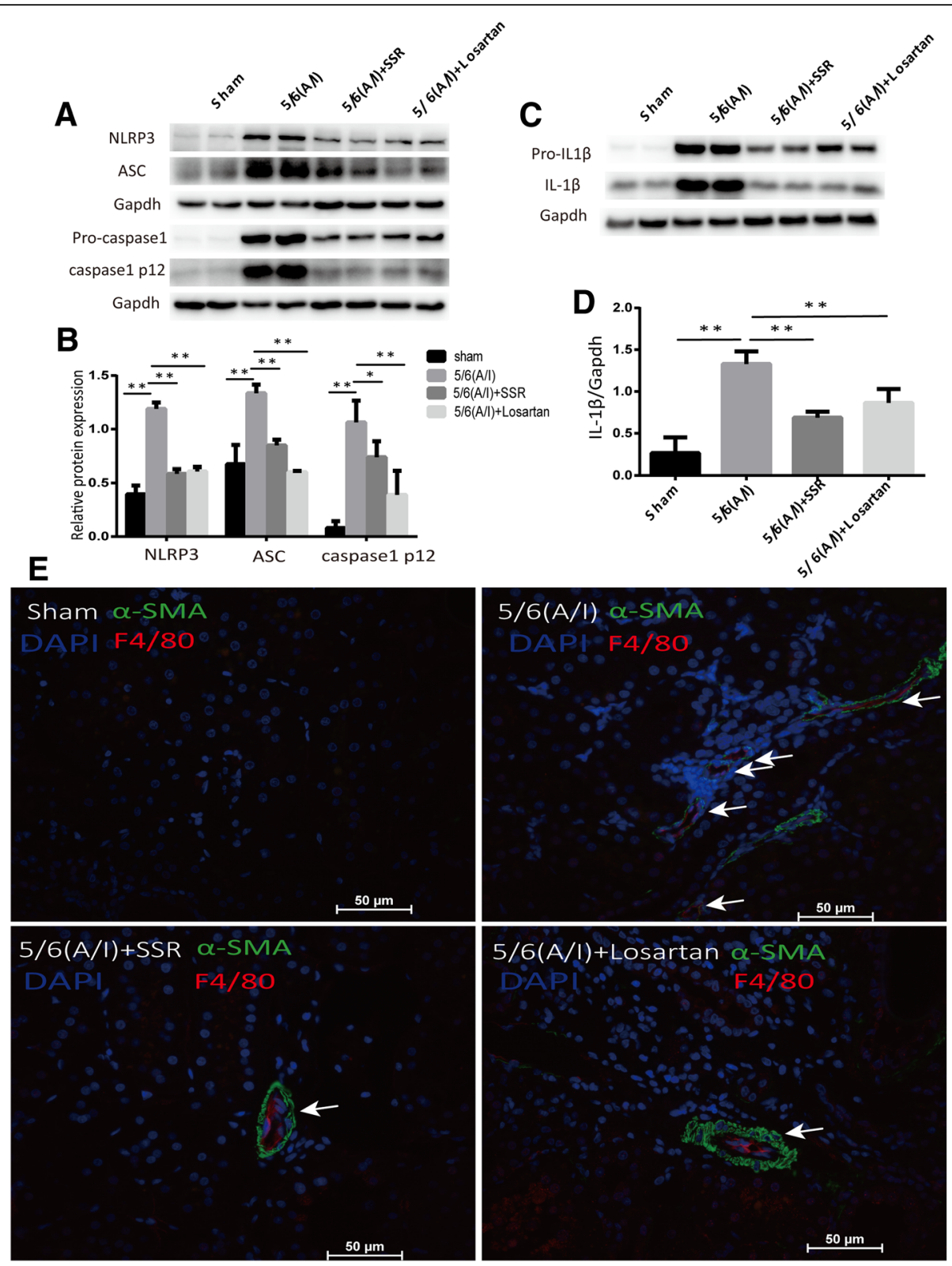

Fig. 2 SSR inhibited NLRP3 inflammasome activation and IL-1ßsecretion in 5/6(A/I) rats. (a) The protein expression of NLRP3,ASC, Procaspase 1 and Caspase-1 p12 was determined by immunoblotting. (b) Western blot quantification of NLRP3, ASC, and Caspase-1 p12 levels. $(n=6)(\mathbf{c})$ The protein levels of Pro-IL1 $\beta$ and IL1 $\beta$ were determined by immunoblotting. (d) The ratio of IL-1 3 to GAPDH protein was determined. $(n=6)$ (e) Representative images of IF staining for $a-S M A$ and F4/80. Original magnification, $\times 400$. Values are mean \pm SD. ${ }^{*} P<0.05,{ }^{* *} P<0.01$

group,which was determined by immunoblotting for the 17-KD cytokine. (Fig. 2(c) and (d)).

To determine whether the tubular injury and renal interstitial fibrosis are associated with inflammatory response,we mainly probed the position of $\mathrm{F} 4 / 80$ positive macrophages and myofibroblast marker $\alpha$-SMA using immunofluorescence double staining. Our results presented the positional correlation between F4/80 and $\alpha$-SMA (Fig. 2e). In addition,the model group reciving 5/
6(A/I) operation displayed increased expression of F4/80 and $\alpha$-SMA proteins compared with sham-operated group. Conversely,SSR dramatically ameliorated these expression of F4/80 and $\alpha$-SMA (Fig. 2e). Taken together,our results revealed SSR effectively inhibited NLRP3/ASC/Caspase-1/IL-1 $\beta$ cascade and decreased inflammatory infiltration in the remnant kidneys,whcih may be associated with alleviated renal injury and interstitial fibrosis. 
SSR reduced Smad3 acetylation in 5/6(A/I) rats by regulating Sirt1/Smad3 deacetylation pathway

Previous reports have shown that Sirt1 could mediate a variety of cellular responses by deacetylating lysine residues such as p53, NF-kB, and PGC-1 $\alpha$ [25-27]. In this study, we first measured the expression of Sirt1 protein. As shown in Fig. 3(a), (b), (c)and (d),compared wtih sham-operated group,immunoblotting and IHC staining showed that injury to kidney decreased the expression of Sirt1 protein,whereas treatment with SSR markedly

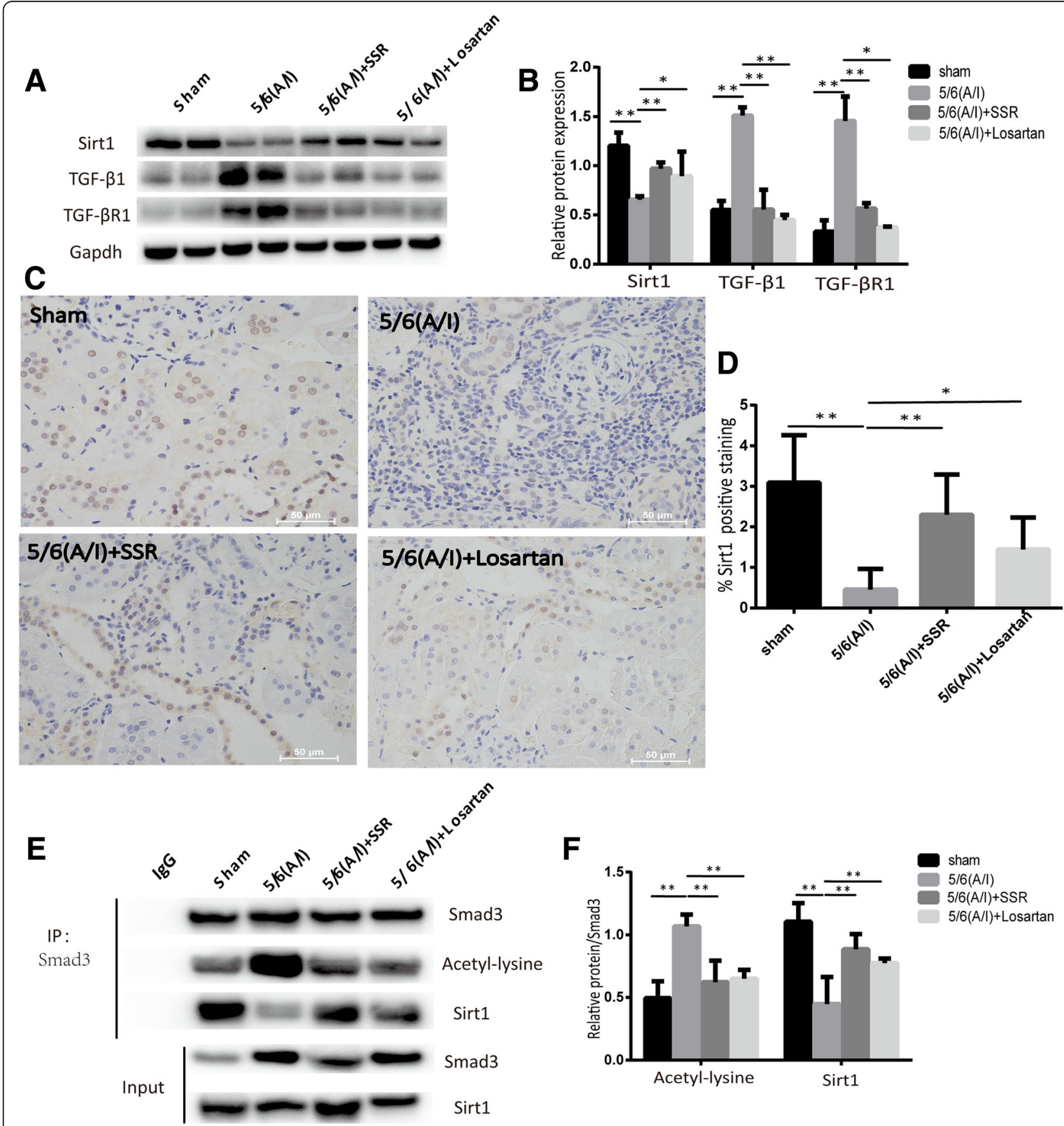

Fig. 3 SSR reduced Smad3 acetylation in 5/6(A/I) rats by regulating Sirt1/Smad3 deacetylation pathway. (a) The protein levels of Sirt1,TGF- $\beta 1$ and TGF- $\beta$ R1 were determined by immunoblotting. (b) The ratio of Sirt1,TGF- $\beta 1$ and TGF- $\beta R 1$ to GAPDH protein was calculated. $(n=6)(\mathbf{c})$ Representative images of IHC staining for Sirt1. $400 \times$ magnification. (d) Quantitative analysis of Sirt1 positive area( $n=4)($ e) Immunoprecipitation and western blot analysis of Sirt1 and Smad3 acetylation in each group. (f) The ratio of Sirt1 and acetylated Smad3 to precipitated Smad3 protein was calculated. $(n=4)$ Values are mean \pm SD. ${ }^{*} P<0.05,{ }^{* *} P<0.01$ 
preserved Sirt1 expression (Fig. 3(a), (b), (c)and (d)).IHC staining confirmed Sirt1 is mainly located in the nucleus (Fig. 3c).Moreover,immunoblotting examination showed expression of typeITGF- $\beta$ R (TGF- $\beta$ R 1 ) and TGF- $\beta 1$ proteins was increased in the $5 / 6(\mathrm{~A} / \mathrm{I})$ model group as compared to that in the sham-operated group (Fig. 3(a) and (b)).Meanwhile, SSR suppressed the expression of TGF- $\beta 1$ and TGF- $\beta R 1$ proteins compared with model group.

In addition, to assess the levels of acetylated Smad3,Smad3 was immunoprecipitated and the acetyl group was determined by an anti-acetyl antibody. As shown in Fig. 3(e) and (f),we observed that the acetylation levels of Smad3 increased in 5/6(A/I) rats and decreased in the rats treated with SSR.Furthermore,co-immunoprecipitation and Western blot studies revealed a direct interaction between Smad3 and Sirt1.The Smad3-Sirt1 binding was reduced in model group, which could be associated with increased levels of acetylated Smad3 (Fig. 3(e) and (f)).Conversely,SSR significantly increased the binding of Sirt1 and Smad3 and reduced acetylation levels of Smad3 (Fig. 3(e) and (f)).These data strongly suggest that renal protective effect of SSR may be associated with Sirt1/Smad3 deacetylation pathway.

\section{Discussion}

In the present study, we found that SSR could significantly suppress progressive activation of NLRP3/ASC/ Caspase-1/IL-1 $\beta$ cascade and up-regulate Sirt1/Smad3 deacetylation pathway in the $5 / 6(\mathrm{~A} / \mathrm{I})$ rat model. These changes could inhibit the expression of Col-III,the ECM protein,as well as E-cadherin,the epithelial cell marker. Together, these results identified that SSR significantly attenuated renal injury and fibrosis in the CRF model. In addition, our results showed that losartan, an angiotensin receptor blocker, seemed to be more effective than SSR in attenuating renal injury and fibrosis, inhibiting activation of NLRP3 complex and down-regulating the expression of TGF- $\beta 1$ and TGF- $\beta$ R 1 proteins. Losartan may significantly reduce the increase of angiotensin II (Ang-II) induced by ischemia because $5 / 6(\mathrm{~A} / \mathrm{I})$ model is a typical intrarenal ischemia model [22, 28]. However, the specific mechanisms need further research.

Nonmicrobial (sterile) inflammatory response is an important characteristic of many CKDs [29].NLRP3 inflammasome is known to be activated by a variety of nonmicrobial signals, and therefore, NLRP3-dependent inflammation could be an attractive candidate as a mediator of the inflammatory component observed in CKD [8]. Previous studies reported NLRP3 complex was involved in the progression of kidney diseases in UUO model [9],ischemia/reperfusion injury (IRI) [30], and diabetic nephropathy [31] etc. and traditional Chinese herbs had obvious inhibition on it. Ren et al. [32]. reported Coptidis Rhizoma could attenuate early renal injury in obesity-related glomerulopathy (ORG) rats and the mechanisms were associated with the inhibition of NLRP3 inflammasome. Chang et al. [33]. demonstrated that rhein suppressed the NLRP3 inflammasome and macrophage activation in urate crystal-induced gouty inflammation. In addition, many active ingredients of SSR, such as magnesium lithospermate B from Salvia miltiorrhiza bunge, ferulic acid from Angelica sinensis,icariin from Epimedium, have shown anti-inflammatory effects in kidney diseases [34-36]. The activation of NF-kB signaling pathway stimulated renal inflammation in different models [37, 38]. Meanwhile,many reports revealed that NF- $\mathrm{KB}$ signaling pathway played a prime role in NLRP3 inflammasome activation [39, 40]. We have investigated the activity of NF- $\mathrm{KB}$ by phosphorylation of p65, its translocation to nucleus. Our results showed that SSR significantly reduced the nuclear translocation of p65 in 5/6(A/I) model [20]. These results prompted us to further study the possible role of NLRP3 inflammasome in 5/6(A/I) model, as well as the mechanisms of SSR attenuating renal fibrosis. Our results showed progressive activation of NLRP3/ASC/Caspase-1 cascade in CRF rat model,which led to the secretion of mature IL-1 $\beta$ pro-inflammatory factor. Inflammation initiates and sustains renal tubulointerstitial fibrosis [41],therefore,we probed colocalization of F4/80,a representative hallmark of macrophages,and $\alpha$-SMA,a marker of fibroblast activation using immunofluorescence double staining. Our data showed that the increased F4/80 and $\alpha$-SMA co-localized in the renal tubular areas. Conversely, activation of NLRP3 inflammasome analysed by immunoblotting and image analysis of IF staining showed significant down-regulation in treatment with SSR,verifying that one of the mechanisms by which SSR attenuates renal injury and delays the progression of renal interstitial fibrosis could be to inhibit inflammatory response induced by activation of NLRP3 complex.

Chronic inflammation is integrally associated with progressive CKD, as cytokines (TGF- $\beta 1$, IL-18, IL-1 $\beta$,etc.) produced by infiltrating leukocytes such as macrophages contribute significantly to renal injury and fibrosis [42].TGF- $\beta 1$ promotes tissue fibrosis through the Smad3 pathway. While phosphorylation regulates Smad3 function, acetylation/de-acetylation of specific lysine residues in Smad3 has been shown to regulate TGF- $\beta 1$ signaling by non-canonical signaling pathways [15]. In vitro,previous studies confirmed Smad3 could be acetylated in response to TGF- $\beta 1$ stimulation [43, 44]. Recently, the lysine deacetylase sirtuin 1 (SIRT1) has been shown to exert renal protective effects [45]. Huang et al. [46]. reported that resveratrol, an activator of the Sirt1, ameliorated renal fibrosis by inhibiting the TGF- $\beta 1 /$ Smad3 pathway. Here, we investigated whether pharmacological activation of Sirt1 would induce renal 
protection in 5/6(A/I) model. In this study, we showed the binding of Sirt1 with Samd3 and inhibition of this binding was associated with increased levels of acetylated Smad3. SSR down-regulated the acetylation levels of Smad3 by activating Sirt1 deacetylation pathway. These data highlight that renal Sirt1/Smad3 deacetylation pathway could be an antifibrotic factor and a potential therapeutic strategy for CKD.

\section{Conclusions}

In summary, we demonstrated that SSR could mediate renal protection by inhibiting NLRP3/ASC/caspase-1/ IL-1 $\beta$ pathway and, furthermore,strengthen the antifibrotic effects by up-regulating Sirt1/Smad3 deacetylation pathway in 5/6th (A/I) model rats with CRF.

\section{Abbreviations \\ 5/6 (A/I): 5/6ablation/ infarction; Ang-II: Angiotensinll; BUN: Blood urea nitrogen; CKD: Chronic kidney disease; Col-I: Collagen-l; Col-III: Collagen-III; CRF: Chronic renal failure; ECM: Extracellular matrix; eGFRs: Evaluated glomerular filtration rates; FN: Fibronectin; GS: Glomerulosclerosis; IF: Immunofluorescence; IHC: Immunohistochemical; IL: Interleukin; IRI: Ischemia/reperfusion; ORG: Obesity-related glomerulopathy; PAS: Periodic acid-Schiff; Scr: Serum creatinine; SD: Sprague-Dawley; Sirt1: Sirtuin1; SSR: ShenShuai IIRecipe; TCM: Traditional Chinese medicine; TGF $\beta$ - 1: Transforming growth factor $\beta-1$; TGF- $\beta$ R1: TypelTGF- $\beta R$; $\alpha$-SMA: $\alpha$-smooth muscle actin}

\section{Acknowledgements}

Not applicable.

\section{Funding}

This study was supported by the National Natural Science Foundation of China General Projects (81573946), Scientific Research Foundation of Science and Technology Commission of Shanghai Municipal Government (16401931700), Shanghai Three Year Project of Traditional Chinese Medicine(ZY(2018-2020)-FWT X-7005). The funding bodies did not participate in the design of the study; collection, analysis, and interpretation of data; and writing of the manuscript.

\section{Availability of data and materials}

The datasets used and/or analyzed during the current study are available from the corresponding author on reasonable request.

\section{Authors' contributions}

CW designed the study and participated in data analysis and interpretation.MW and LYY finalized the experimental work, interpreted the results and prepared Figs.MW wrote the paper, CW and JY edited and revised. All authors read and approved the final version of manuscript.

\section{Ethics approval and consent to participate}

The animal procedures were approved by the Animal Experiment Ethics Committee of Shanghai University of Traditional Chinese Medicine in accordance with the principles outlined in the NIH Guide for the Care and Use of Laboratory Animals.

\section{Consent for publication}

Not applicable.

\section{Competing interests}

The authors declare that they have no competing interests.

\section{Publisher's Note}

Springer Nature remains neutral with regard to jurisdictional claims in published maps and institutional affiliations.

\section{Author details}

'Department of Nephrology, Shuguang Hospital Affiliated to Shanghai University of Traditional Chinese Medicine, Shanghai 201203, China. ${ }^{2}$ Key Laboratory of Liver and Kidney Diseases,Ministry of Education, Shanghai University of Traditional Chinese Medicine, Shanghai 201203, China. ${ }^{3}$ TCM institute of kidney disease, Shanghai University of Traditional Chinese Medicine, Shanghai 201203, China. ${ }^{4}$ Shanghai Key Laboratory of Traditional Chinese Clinical Medicine, Shanghai University of Traditional Chinese Medicine, Shanghai 201203, China.

Received: 19 February 2019 Accepted: 15 May 2019

Published online: 22 May 2019

\section{References}

1. Mutsaers HA, Olinga P. Editorial: organ fibrosis: triggers, pathways, and cellular plasticity. Front Med. 2016;3:55.

2. Ene-lordache B, Perico N, Bikbov B, Carminati S, Remuzzi A, Perna A, Islam N, Bravo Rodolfo F, Aleckovic-Halilovic M, Zou H, Zhang LX, Gouda Z, Tchokhonelidze I, Abraham G, Mahdavi-Mazdeh M, Gallieni M, Codreanu I, Togtokh A, Sharma SK, Koirala P, Uprety S, Ulasi I, Remuzzi G. Chronic kidney disease and cardiovascular risk in six regions of the world (ISN-KDDC): a cross-sectional study. Lancet Glob Health. 2016:4:e307-19.

3. Eddy AA. Overview of the cellular and molecular basis of kidney fibrosis. Kidney Int Suppl. 2014:4:2-8.

4. Djudjaj S, Boor P. Cellular and molecular mechanisms of kidney fibrosis. Mol Asp Med. 2018:1-21.

5. Li ZL, Lv LL, Tang TT, Wang B, Feng Y, Zhou LT, Cao JY, Tang RN, Wu M, Liu H, Crowley SD, Liu BC. HIF-1ainducing exosomal microRNA-23a expression mediates the cross-talk between tubular epithelial cells and macrophages in tubulointerstitial inflammation. Kidney Int. 2019:95:388-404.

6. Ting JY, Lovering RC, Alnemri ES, Bertin J, Boss JM, Davis BK, Flavell RA, Girardin SE, Godzik A, Harton JA, Hoffman HM, Hugot JP, Inohara N, Mackenzie A, Maltais $\sqcup$, Nunez G, Ogura Y, Otten LA, Philpott D, Reed JC, Reith W, Schreiber S, Steimle V, Ward PA. The NLR gene family: a standard nomenclature. Immunity, 2018:28:285-7.

7. He Y, Hara H, Núñez G. Mechanism and regulation of NLRP3 InflammasomeActivation. Trends Biochem Sci. 2016:41:1012-21.

8. Song N, Liu ZS, Xue W, Bai ZF, Wang QY, Dai J, Liu X, Huang YJ, Cai H, Zhan XY, Han QY, Wang H, Chen Y, Li HY, Li AL, Zhang XM, Zhou T, Li T. NLRP3 phosphorylation is an essential priming event for Inflammasome activation. Mol Cell. 2017;68(1):185-97.

9. Vilaysane A, Chun J, Seamone ME, Wang WJ, Chin R, Hirota S, Li Y. ClarkSA,Tschopp J, Trpkov K, Hemmelgarn BR, Beck PL, Muruve DA. The NLRP3 inflammasome promotes renal inflammation and contributes to CKD. J Am Soc Nephrol. 2010;21:1732-44.

10. Zhuang Y, Yasinta M, Hu C, Zhao M, Ding G, Bai M, Yang LY, Ni JJ, Wang R, Jia ZJ, Huang SG, Zhang AH. Mitochondrial dysfunction confers albumininduced nlrp3 inflammasome activation and renal tubular injury. Am J Physiol Renal Physiol. 2015;308(8):F857-66.

11. Ludwig-Portugall I, Bartok E, Dhana E, Evers BG, Primiano MJ, Hall JP,Franklin,BS,Knolle PA, Hornung V, Hartmann G, Boor P, Latz E, Kurts C. An NLRP3-specific inflammasome inhibitor attenuates crystal-induced kidney fibrosis in mice. Kidney Int2016; 90:525-539.

12. Ignotz RA, Massagué J. Transforming growth factor-beta stimulates the expression of fibronectin and collagen and their incorporation into the extracellular matrix. J Biol Chem. 1986;261:4337-45.

13. Roberts AB, Russo A, Felici A, Flanders KC. Smad3: a key player in pathogenetic mechanisms dependent on TGF-beta. Ann N Y Acad Sci. 2003; 995:1-10.

14. Roberts AB, Tian F, Byfield SD, Stuelten C, Ooshima A, Saika S, Flanders KC. Smad3 is key to TGF-beta-mediated epithelial-to-mesenchymal transition, fibrosis, tumor suppression and metastasis. Cytokine Growth Factor Rev. 2006:17:19-27.

15. Li J, XI Q, Ricardo SD, Bertram JF, Nikolic-Paterson DJ. Resveratrol inhibits renal fibrosis in the obstructed kidney: potential role in deacetylation of Smad3. Am J Pathol. 2010:177:1065-71.

16. Hao CM, Haase VH. Sirtuins and their relevance to the kidney. J Am Soc Nephrol. 2010:21:1620-7.

17. He Wj WYY, Zhang MZ, You L, Davis $L S$, Fan $H$, Yang HC, Fogo AB, Zent $R$, Harris RC, Breyer MD, Hao CM. Sirt1 activation protects the mouse renal medulla from oxidative injury. J Clin Invest. 2010;120:1056-68. 
18. Li JW, Liu XF, Chen HZ, Sun ZY, Chen H, Wang L, Sun XH, Li XQ. Multitargeting chemoprevention of Chinese herb formula Yanghe Huayan decoction on experimentally induced mammary tumorigenesis. BMC Complement Altern Med. 2019;19:48-62.

19. Xu YY, He Z, Zhou Y, Yang J, Wang C. Effects of the meliorated renal FailureDecoction combined with Western medicine on renal function and renal perfusion in primary chronic kidney disease 3 or 4 stage patients. J Tradit ChinMed. 2018;59(17):1480-4 in Chinese.

20. Yang J, Zhu TT, Wang C. Effects of Shenshuai IIDecoction on NF-kB /TNF-a signaling pathway expressions in renal tissues of chronic renal failure rats. Chin J Inform on TCM. 2018;25(8):58-62 in Chinese.

21. Wang M, Yang J, Zhou Y, Wang C. ShenShuai II recipe attenuates apoptosis and renal fibrosis in chronic kidney disease by increasing renal blood flow and improving oxygen consumption. Evid Based Complement Alternat Med. 2018:1-8.

22. Deng AH, Tang T, Singh P, Wang C, Satriano J, Thomson SC, Blantz RC. Regulation of oxygen utilization by angiotensin II in chronic kidney disease. Kidney Int. 2009;75:197-204.

23. Wang M, Han WZ, Zhang M, Fang WY, Zhai XR, Guan SF, Qu XK. Long-term renal sympathetic denervation ameliorates renal fibrosis and delays the onset of hypertension in spontaneously hypertensive rats. Am J Transl Res. 2018;10:4042-53.

24. Lv W, Booz GW, Wang YG, Fan F, Roman RJ. Inflammation and renal fibrosis: recent developments on key signaling molecules as potential therapeutic targets. Eur J Pharmacol. 2018;820:65-76.

25. Yuan F, Xie Q, Wu J, Bai Y, Mao B, Dong Y, Bi W, Ji G, Tao W, Wang Y, Yuan Z. MST1 promotes apoptosis through regulating Sirt1-dependent p53 deacetylation. J Biol Chem. 2011;286:6940-5.

26. Yeung F, Hoberg JE, Ramsey CS, Keller MD, Jones DR, Frye RA, Mayo MW. Modulation of NFkappaB-dependent transcription and cell survival by the SIRT1 deacetylase. EMBO J. 2004;23:2369-80.

27. Amat R, Planavila A, Chen SL, Iglesias R, Giralt M, Villarroya F. SIRT1 controls the transcription of the peroxisome proliferator-activated receptor-gamma co-activator-1alpha (PGC-1alpha) gene in skeletal muscle through the PGC 1alpha autoregulatory loop and interaction with MyoD. J Biol Chem. 2009; 284:21872-80.

28. Manotham K, Tanaka T, Matsumoto M, Ohse T, Miyata T, Inagi R, Kurokawa K, Fujita T, Nangaku M. Evidence of tubular hypoxia in the early phase in the remnant kidney model. J Am Soc Nephrol. 2004;15:1277-88.

29. Ricardo SD, van GH, Eddy AA. Macrophage diversity in renal injury and repair. J Clin Invest. 2008;118:3522-30.

30. Kim HJ, Lee DW, Ravichandran K, O-Keys D, Akcay A, Nguyen Q, He ZB, Jani A, Ljubanovic D, Edelstein CL. NLRP3 inflammasome knockout mice are protected against ischemic but not cisplatin-induced acute kidney injury. J Pharmacol Exp Ther. 2013;346:465-72.

31. Chen K, Zhang J, Zhang W, Yang J, Li K, He Y. ATP-P2X4 signaling mediates NLRP3 inflammasome activation: a novel pathway of diabetic nephropathy. Int J Biochem Cell Biol. 2013;45:932-43.

32. YI R, Dk W, Lu F, Zou X, Lj X, Wang KF, Huang WY, Su H, Zhang C, Gao Y, Dong H. Coptidis Rhizoma inhibits NLRP3 inflammasome activation and alleviates renal damage in early obesity-related glomerulopathy. Phytomedicine. 2018;49:52-65.

33. Chang WC, Chu MT, Hsu CY, Wu YJ, Lee JY, Chen TJ, Chung WH, Chen DY, Hung SL. Rhein, an Anthraquinone drug, suppresses the NLRP3 Inflammasome and macrophage activation in urate crystal-induced gouty inflammation. Am J Chin Med. 2019;47:135-51.

34. Park CH, Shin SH, Lee EK, Kim DH, Kim MJ, Roh SS, Yokozawa T, Chung $\mathrm{H}$. Magnesium Lithospermate B from Salvia miltiorrhiza Bunge ameliorates aging-induced renal inflammation and senescence via NADPH oxidase-mediated reactive oxygen generation. Phytother Res. 2017;31:721-8.

35. Chowdhury S, Ghosh S, Das A, Sil P. Ferulic acid protects hyperglycemiainduced kidney damage by regulating oxidative insult, inflammation and autophagy. Front Pharmacol. 2019;10:1-24.

36. Chen HA, Chen CM, Guan SS, Chiang CK, Wu CT, Liu SH. The antifibrotic and anti-inflammatory effects of icariin on the kidney in a unilateral ureteral obstruction mouse model. Phytomedicine. 2019;59:1-7.

37. Henke N, Schmidt-Ullrich R, Dechend R, Park JK, Qadri F, Wellner M, Obst M, Gross V, Dietz R, Luft F, Scheidereit C, Muller D. Vascular endothelial cellspecific NF-kappaB suppression attenuates hypertension-induced renal damage. Circ Res. 2007;101:268-76.
38. Kim J, Ha I, Hwang C, Lee YJ, Kim J, Yang SH, Kim YS, Cao YA, Choi S, Park WY. Gene expression profiling of anti-GBM glomerulonephritis model: the role of NF-kappaB in immune complex kidney disease. Kidney Int. 2004;66: 1826-37.

39. Bauernfeind F, Horvath G, Stutz A, Alnemri E, MacDonald K, Speert D, Fernandes-Alnemri T, Wu J, Monks B, Fitzgerald K, Hornung V, Latz E. Cutting edge: NF-kappaB activating pattern recognition and cytokine receptors license NLRP3 inflammasome activation by regulating NLRP3 expression. J Immunol. 2009;183:787-91.

40. Lamkanfi M, Dixit V. Mechanisms and functions of inflammasomes. Cell. 2014:157:1013-22

41. Zhang Y, XI S, Zou FF, Xu TJ, Pan PH, Hu CP. Toll-like receptor-4 deficiency alleviates chronic intermittent hypoxia-induced renal injury, inflammation, and fibrosis. Sleep Breath. 2018:1-12.

42. Wang WJ, Wang XY, Chun J, Vilaysane A, Clark S, French G, Bracey NA, Trpkov K, Bonni S, Duff HJ, Beck PL, Muruve DA. Inflammasomeindependent NLRP3 augments TGF- $\beta$ signaling in kidney epithelium. J Immunol. 2013;190:1239-49.

43. Simonsson M, Kanduri M, Grönroos E, Heldin CH, Ericsson J. The DNA bindingactivities of $\mathrm{Smad} 2$ and $\mathrm{Smad} 3$ are regulated by coactivatormediated acetylation. J Biol Chem. 2006;281:39870-80.

44. Inoue $Y$, Itoh $Y$, Abe K, Okamoto T, Daitoku H, Fukamizu A, Onozaki K, Hayashi $\mathrm{H}$. Smad3 is acetylated by p300/CBP to regulate its transactivation activity. Oncogene. 2007;26:500-8.

45. Kitada M, Takeda A, Nagai T, Ito H, Kanasaki K, Koya D. Dietary restriction ameliorates diabetic nephropathy through anti-inflammatory effects and regulation of the autophagy via restoration of Sirt1 in diabetic Wistar fatty (fa/fa) rats: a model of type 2 diabetes. Exp Diabetes Res. 2011:1:11.

46. Huang $X Z$, Wen DH, Zhang $M$, Xie QH, Ma L, Guan Y, Ren YH, Chen J, Hao CM. Sirt1 activation ameliorates renal fibrosis by inhibiting the TGF- $\beta / S$ mad3 pathway. J Cell Biochem. 2014;115:996-1005.
Ready to submit your research? Choose BMC and benefit from:
- fast, convenient online submission
- thorough peer review by experienced researchers in your field
- rapid publication on acceptance
- support for research data, including large and complex data types
- gold Open Access which fosters wider collaboration and increased citations
- maximum visibility for your research: over $100 \mathrm{M}$ website views per year
At BMC, research is always in progress.
Learn more biomedcentral.com/submissions 